\title{
Pródromos de transtornos de humor e suporte familiar em adolescentes escolares
}

\author{
Prodromal mood disorders and family support in school adolescents
}

\author{
Mercedes da Silva Strider', Ingrid D'Avila Francke', Tiago da Rocha Ribeiro \\ e Felipe Ornell ${ }^{4}$
}

Resumo: Os transtornos mentais em crianças e adolescentes atingem uma taxa de prevalência entre $10 \%$ e $20 \%$ e geralmente apresentam-se com problemas na esfera emocional e de conduta, representando prejuízos, como perdas nas relações sociais, familiares e escolares. Este trabalho teve o objetivo de identificar a prevalência de pródromos de transtornos de humor e a percepção de suporte social em adolescentes escolares. Como método utilizouse a pesquisa transversal, quantitativa, com objetivo exploratório e de alcance correlacional. A amostra de 135 alunos, com idade entre 12 e 17 anos respondeu a Escala de Percepção do Suporte Social - Adolescente (EPSUS-Ad), Self-Reporting Questionnaire (SRQ-20) e Questionário Sociodemográfico. Após as análises estatísticas concluiu-se que as variáveis discutidas neste estudo indicam um coeficiente de correlação linear positiva forte entre "presença de pródromos para transtorno de humor" e "baixa percepção de suporte social", com $r=0,9863$. Assim, sugere-se atenção para políticas públicas de cuidado à saúde mental de crianças e adolescentes, que garantam acesso universal e equânime, a fim de minimizar o sofrimento, prevenir agravos e promover a saúde.

Palavras-chave: Pródromos; Suporte familiar; Transtornos de humor.
Abstract: Mental disorders in children and adolescents reach a prevalence rate between 10\% and $20 \%$ and usually present themselves with emotional and behavioral problems, representing losses in social, family, and school relationships. This study aimed to identify the prevalence of prodromal mood disorders and the perception of social support in adolescent students. The method used was a transversal, quantitative research, with exploratory and correlational objectives. The sample of 135 students, aged between 12 and 17 years old, answered the Scale of Perceived Social Support - Adolescent (EPSUS-Ad), Self-Reporting Questionnaire (SRQ-20) and Sociodemographic Questionnaire. After the statistical analyses it was concluded that the variables discussed in this study indicate a strong positive linear correlation coefficient between "presence of prodromes for mood disorder" and "low perception of social support", with $r=0.9863$. Thus, attention is suggested for public policies of care for the mental health of children and adolescents, which guarantee universal and equitable access, in order to minimize suffering, prevent worsening and promote health.

Keywords: Prodromes; Family support; Mood Disorders.

\footnotetext{
1 Psicóloga, Especializanda em Teoria Psicanalítica e Clínica Psicoterápica no CIPT/POA. E-mail: mercedesstrider@gmail.com

2 Psicóloga, Doutora em Saúde Coletiva, Orientadora do Curso de Psicologia Universidade Luterana do Brasil - Campus Guaíba. E-mail: ingrid.francke@ulbra.br

3 Discente do Curso de Psicologia da Universidade Luterana do Brasil - Campus Guaíba. E-mail: tiago.ribeiro@rede.ulbra.br

4 Psicólogo, possui residência em Saúde Mental (ESPRS) e especialização em Dependência Química; Mestre e Doutorando em Psiquiatria e ciências do comportamento (UFRGS). E-mail: fornell@hcpa.edu.br
} 


\section{Introdução}

Atualmente não há um consenso sobre a idade da adolescência, pois a Organização Mundial da Saúde (OMS) define a adolescência entre 10 e 19 anos, e segundo 0 Estatuto da Criança e do Adolescente (ECA) esse período compreende a faixa etária entre 12 e 18 anos (Souza, Barbosa, \& Moreno, 2015). De acordo com Silva, Alvim, Costa, Ramos e Costa (2015), a adolescência é um período vulnerável, pois ocorrem diversas mudanças e transformações características da fase.

Os transtornos mentais em crianças e adolescentes atingem uma taxa de prevalência entre 10\% e 20\% e geralmente apresentam-se com problemas na esfera emocional e de conduta, representando prejuízos, como perdas nas relações sociais, familiares e escolares. Além disso, os transtornos mentais são persistentes, resultando que boa parte dessas pessoas têm algum tipo de comprometimento ao longo da vida adulta. (Lopes et al., 2016).

Os Transtornos do Humor são caracterizados pela perda do senso de controle, perda de energia e interesses, humor alterado, ausência de prazer nas suas atividades, alterações no sono, dificuldades na tomada de decisões, de concentração e pensamento, apetite, presença de baixa autoestima, sentimentos de inutilidade, culpa e pensamentos de morte, sendo que esses sintomas afetam e comprometem a vida do indivíduo em diversos aspectos social e profissional. (Associação Americana de Psiquiatria [APA], 2013; Kaplan \& Sadock, 1993).

Os Transtornos de Humor de maior prevalência são Transtorno Bipolar (TB) eTranstorno Depressivo Maior (TDM). De acordo com a OMS (2016) em seu relatório houve um aumento entre os anos de 2005 e 2015 de 18\% no número de pessoas com depressão. Aliado a isso temos o Transtorno Bipolar que estima-se que 4\% da população mundial sofra e segundo a Associação Brasileira de Transtorno Bipolar (ABTB) (2016), no Brasil a prevalência é de aproximadamente 6 milhões de pessoas.

0 TB apresenta-se com alterações graves de humor, que vão desde ocorrências de humor elevado até períodos depressivos, sendo que estes se alternam e se relacionam com sintomas cognitivos, físicos e comportamentais. Jáno TDM, podemos observar um humor rebaixado e anedonia, alterações de peso, apetite, sono e de comportamento. (Clemente, 2015).

Não há dados conclusivos sobre a prevalência do TB em crianças e adolescentes. Mas em crianças e adolescentes pode apresentar sintomas diferentes dos característicos da idade adulta. As crianças, na maior parte das vezes, apresentam ciclos rápidos com quatro episódios de humor anuais, além das características de alterações bruscas e diárias de humor, presença de sintomatologia depressiva, episódios maníacos em forma de irritabilidade e evolução gradativa e crônica desde a infância (Phillips \& Kupfer, 2013).

De acordo com a OMS (2017), a depressão não acomete apenas adultos, mas também crianças e adolescentes com idade inferior a 15 anos, em um nível mais baixo ao comparado com os adultos, havendo outras formas de apresentação, sendo elas mais indiretas (alterações do comportamento, do sono ou do apetite e dificuldade de concentração). A presença destes sintomas altera também como a criança lida e enfrenta as suas emoçoes, frustrações e representações. Os mesmos autores apontam que, no Brasil, existem poucos estudos epidemiológicos, mas os resultados existentes revelam altos índices de presença de sintomas depressivos entre escolares adolescentes e crianças. Esses estudos contribuem para um melhor levantamento das características relevantes para o diagnóstico de transtorno, principalmente das diferenças de gênero. (Hay, Levin, \& Deterding, 2016; Pinheiro, Sousa, Feitosa, \& Batista, 2017).

Ao se tratar dos sintomas, Baptista, Borges e Serpa (2017) trazem que na infância os Transtornos de Humor se manifestam de forma diferente de acordo com o sexo, onde os meninos com idade entre 8 e 12 anos apresentam maiores dificuldades na resolução de problemas, agressividade, problemas de concentração, pensamentos e ideação suicida, baixa sociabilidade e solidão. Já as meninas na mesma faixa etária apresentam maior ausência de prazer, alterações no apetite, baixa autoestima, desesperança, rebaixamento de humor, sociabilidade reduzida e vontade de chorar.

Ainda sobre a gravidade da presença de Transtornos de Humor na infância e adolescência, Ferrari e Barreto (2018), referem a vulnerabilidade destes jovens quando acometidos por pensamentos mórbidos. Em geral, estes pensamentos eliciam comportamentos suicidas ou de automutilação associadas a alguns fatores estressores que costumam se relacionar a risco aumentado de suicídio, como a perda recente de apoio social, o que inclui separaçōes e perdas afetivas, relação familiar conturbada etc.

0 início das doenças mentais geralmente é gradual; muito raramente começa de forma súbita, sem sintomas precursores [...]. 0 início dos sintomas pode preceder em anos ou até décadas, 0 início da doença. 0 início da doença parece relacionar-se a períodos específicos da vida, especialmente aos relacionados ao desenvolvimento. (Emil Kraepelin, 1909 como citado em Noto, 2014, p. 26).

Em uma tentativa de prevenção ao desenvolvimento de Transtornos de Humor na infância, estudos vêm explorando formas de verificar indicadores da vulnerabilidade à doença. Geralmente, na prática clínica observa-se a existência de um período em que surgem sintomas que indicam uma proximidade ao transtorno, contudo ainda não o suficiente para caracterizar uma ruptura completa com o funcionamento psíquico habitual do indivíduo. 0 período que precede a eclosão de doençass, como a esquizofrenia ou $0 \mathrm{~TB}$, vem sendo denominado pródromo. "0 pródromo de uma doença em geral é definido como a fase de mudanças dinâmicas de comportamento e do estado mental que antecedem o primeiro episódio sindrômico da doença" (Noto, 2014).

A presença de um estado clínico precursor identificável e, portanto, a possibilidade de prevenção, ainda carece de muitos estudos. Além disso as mudanças nos critérios de classificação dos transtornos, segundo o DSM-5 (APA, 2013), tornou esta identificação dependente de uma decisão categórica inespecíica sobre o início dos pródromos destes transtornos. Contudo, alguns estudos retrospectivos mostram, por exemplo, que "pacientes bipolares referem sintomas em estágios tão precoces quanto os dois anos de idade, embora os mesmos sejam muito inespecíficos, tais como distúrbios do sono ou choro excessivo" (Noto, 2014). Ainda segundo este autor:

Ao longo do tempo, com a progressão da psicopatologia e 0 amadurecimento do indivíduo, existe uma propensão para que os sintomas se tornem mais específicos e mais parecidos com 0 TB já instalado. Por exemplo, um estudo de seguimento de quatro anos, encontrou que $38 \%$ das crianças e adolescentes inicialmente diagnosticadas com sintomas subsindrômicos de TB e 25\% das 
diagnosticadas com TB tipo II transitaram para o diagnóstico de TB tipo I durante 0 acompanhamento. (Noto, 2014, p. 27)

No que se refere a suporte social, estudos referem a existência de, pelo menos, três pilares para o desenvolvimento do adolescente, sendo eles oriundos da família, dos amigos e da escola. (Alves \& Dell'Aglio, 2015). Outros autores como Sette, Capitão e Carvalho (2016) e Vieira (2015) também citam que os jovens que apresentaram mais sintomas depressivos tiveram uma menor percepção de suporte familiar/social. Uma vez a família oferecendo os cuidados básicos, além de afeto, atenção, independência, diálogos, acolhimento e autonomia, a mesma é considerada um importante fator protetivo e fonte de suporte, sendo este suporte imprescindível para o crescimento saudável do indivíduo. (Souza \& Baptista, 2017).

A literatura traz diversos autores que abordam que uma boa percepção de suporte familiar é muito importante para o desenvolvimento biopsicossocial da criança e do adolescente, sendo a ausência desse amplamente ligada a sintomas ou transtornos afetivos em crianças, adolescentes e adultos (Gonçalves, Baptista, \& Farcas, 2016). Desta forma, podemos ver a família como sendo o maior fator protetivo, como rede primária para o estabelecimento de interações sociais e fornece a base necessária para formação e preservação da saúde física e psicológica da pessoa, sendo a falta desse suporte um dos principais fatores associados ao desenvolvimento de transtornos mentais. (Souza \& Baptista, 2017).

Perante a importância do referencial exposto até 0 momento, este trabalho se justifica devido à relevância de trazer este tema para discussão junto à comunidade acadêmica e comunidade em geral. Além disso, estes dados devem contribuir para um maior desenvolvimento de estudos para este público, pois atualmente existem poucos estudos e isso reafirma a importância da teoria para oferecer conteúdos teóricos para os profissionais tanto da área da educação, como da área da saúde e pode indicar possíveis necessidades de criação e implantação de projetos de saúde mental comunitários para esses adolescentes. Também se espera que este possa contribuir para a sociedade local e geral sobre a importância da identificação das características/prodrômicas que se manifestam principalmente no ambiente de maior circulação destes jovens, como a escola.

Esta pesquisa teve os objetivos de identificar a prevalência de pródromos de Transtornos de Humor e fatores associados em adolescentes escolares de uma escola da rede pública da cidade de Guaíba, no Rio Grande do Sul; investigar a associação entre a prevalência de pródromos de Transtornos de Humore fatores associados e a percepção de suporte familiar em adolescentes escolares.

\section{Método}

Como metodologia utilizou-se a pesquisa transversal, quantitativa, com objetivo exploratório e de alcance correlacional (Sampieri \& Lucio, 2013). A amostra foi composta por 135 alunos, de ambos os sexos, com idades entre 12 e 17 anos, recrutados em uma escola da rede pública de um município da região metropolitana de Porto Alegre, no Rio Grande do Sul. 0 projeto foi aprovado pelo Comitê de Ética da Universidade Estadual do Rio Grande do Sul (UERGS) sob o CAAE de número 04313418.8.0000.8091.

Os critérios de inclusão foram que a idade seria de 12 até 17 anos, este jovens deveriam ter acesso à internet por meio de aparelhos celulares, tablets e ou computadores, que tiveram capacidade para compreender o Termo de Consentimento Livre Esclarecido (TCLE) e do Termo de Assentimento Livre Esclarecido (TALE), além da capacidade para compreender e responder os questionários, e não houveram critérios de exclusão.

A coleta de dados ocorreu após a anuência da instituição. Todas as recomendações éticas foram cumpridas e todos os participantes acordaram participar da pesquisa, o que foi formalizado pela assinatura do TCLE e do TALE. A aplicação dos questionários foi realizada por alunos do curso de psicologia treinados previamente.

Informações sociodemográficas foram coletadas por um questionário desenvolvido pelos pesquisadores com questões sobre sexo, idade, ano de escolaridade e como considera a sua situação econômica.

A percepção de suporte social foi ponderada pela Escala de Percepção do Suporte Social, versão Adolescente (EPSUS-Ad), este instrumento permite a ponderação de escores de suporte em baixo, médio baixo, médio alto e alto. (Baptista \& Cardoso, 2011).

A investigação de sintomas físicos e psicoemocionais ocorreu a partir da aplicação da versão adaptada do Self-Reporting Questionnaire (SRQ-20), validada para o Brasil. 0 questionário é composto de 20 questões do tipo sim/não, das quais quatro são sobre sintomas físicos e 16, sobre distúrbios psicoemocionais. (Harding et al., 1980).

Os dados foram digitados no Google Forms e posteriormente exportados e analisados por meio do programa estatístico Statistical Package for Social Science for Windows, versão 18 (SPSS). As associações entre as variáveis categóricas foram apresentadas por frequência absoluta e relativa e investigadas pelo teste de qui-quadrado, adotando-se a significância de 5\%. Variáveis quantitativas foram apresentadas por média e desvio padrão e as diferenças entre grupos foram testadas pelo teste T de Student quadrado e foi adotada a significância de 5\%.

\section{Resultados e discussão}

Participaram desta pesquisa 135 adolescentes estudantes, sendo 59 do sexo masculino (43,70\%) e 76 do sexo feminino (56,30\%), com idade variando entre 12 e 17 anos, sendo a média de idade 15,06 anos (d.p.1,138), agrupados em três níveis socioeconômicos, ruim (10 - 7,4\%), médio (79 $58,5 \%)$ e bom (46 - 34,1\%), e 30 alunos (22,2\%) estão matriculados no ensino fundamental, enquanto 105 (77,8\%) estão matriculados no ensino médio, sendo essa uma escola da rede pública do município de Guaíba no estado do Rio Grande do Sul.

As variáveis discutidas neste estudo foram submetidas a análises bivariadas de correlação e indicam um coeficiente de correlação (r) entre as variáveis "presença de pródromos para Transtorno de Humor" e "baixa percepção de suporte social" de $r=0,9863$.

Isso significa uma correlação linear positiva e forte, pois quanto mais próxima de 1 mais forte a correlação, conforme ilustrado na Figura 1. 
Figura 1. Correlação de pródromos x percepção de baixo suporte

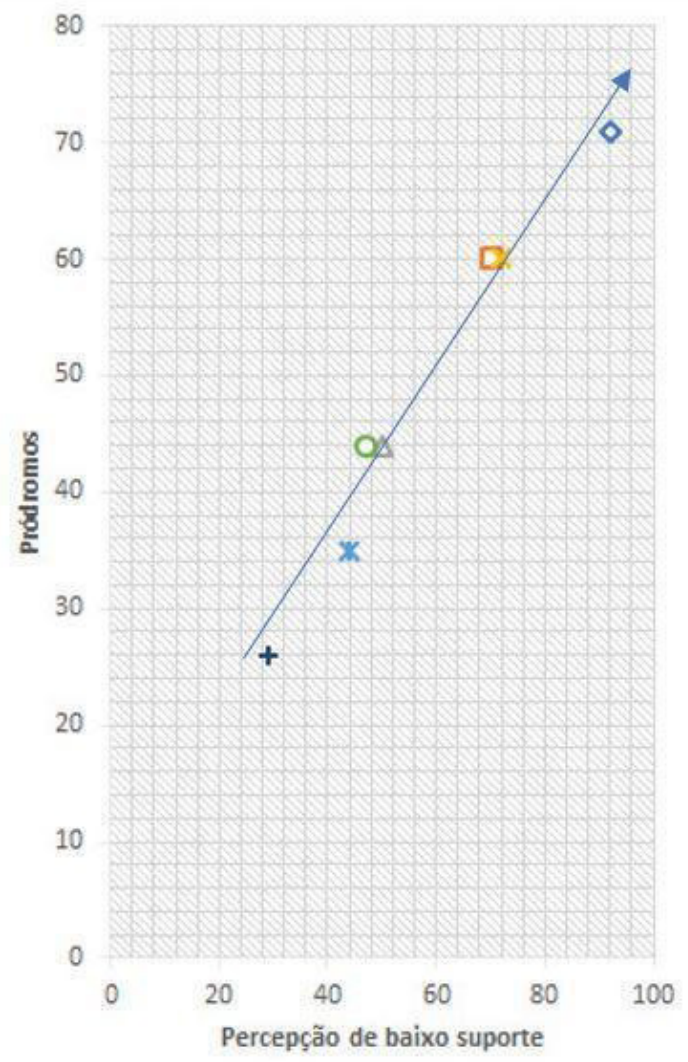

- Sente-se nervoso(a), tenso(a) ou preocupado(a)?

口 Tem se sentido triste ultimamente?

$\Delta$ Tem chorado mais do que de costume?

* Tem perdido o interesse pelascoisas?

x É incapaz de desempenhar um papel útil em suavida?

- Você sesente uma pessoa inútil, sem préstimo?

+ Tem tido ideia de acabar com avida?

- Linear (Sente-se nervoso(a), tenso(a) ou preocupado(a)?)

Fonte: elaborado pelos autores

Analisando as questões do SRQ que indicam possíveis pródromos de transtornos mentais, conforme a Figura 2.

Figura 2. Quantidade de pródromos e a percepção de suporte de baixo com adolescentes

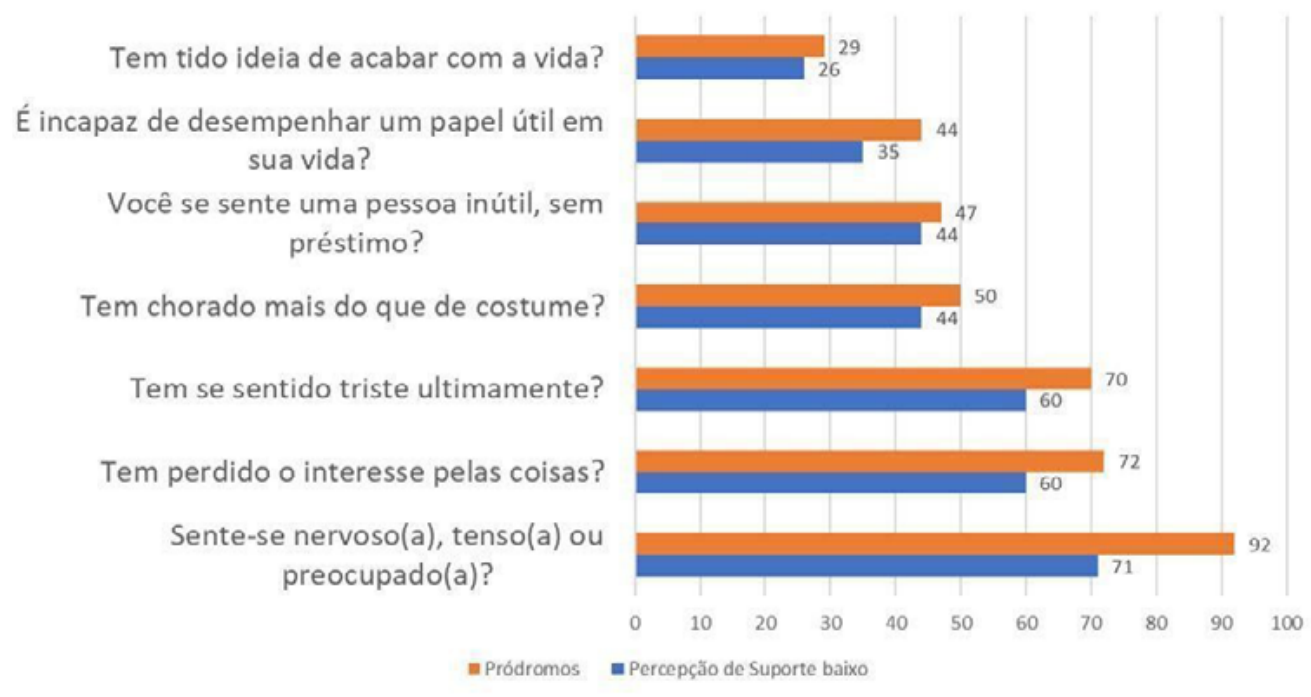

Fonte: elaborado pelos autores

Os resultados individuais apontam que 70 dos 135 adolescentes, ou seja, 53,0\% da amostra tem se sentido triste ultimamente, dos quais 85,7\% possui percepção de suporte baixo e 14,3\% possui percepção de suporte alto. Além disso,
50 dos 135 adolescentes, ou seja, 37,0\% da amostra tem chorado mais do que de costume, dos quais $88,0 \%$ possui percepção de suporte baixo e $12 \%$ possui percepção de suporte alto. Demonstrando uma correlação significativa entre as variáveis. 
Apesar da tristeza ser um indicativo considerável, sua existência por si só não implica em um diagnóstico de transtorno depressivo (Cruvinel \& Boruchovitch, 2014). A tristeza não dura mais que horas e dias e consiste geralmente na resposta às situações de perda, derrota, desapontamento e outras adversidades. Geralmente não causa prejuízo funcional e não é acompanhada de sintomas somáticos importantes.

Segundo James, Reddy, Ellahebokus, Sewpaul e Naidoo (2017), os comportamentos auto prejudiciais pertinentes na adolescência, comumente tidos como resposta de rebeldia, podem, de fato, ter associação com sentimento de tristeza ou desesperança. Demais estudos apontam que adolescentes tendem a valorar mais os afetos negativos e associam com maior intensidade os eventos estressantes com os seus efeitos negativos, podendo gerar uma desregulação emocional (Assumpção, Teodoro, Maltoni,\& Neufeld, 2017).

Segundo Braga e Dell'Aglio (2013), adolescentes argumentam ausência de amigos e queixam-se da falta de al guém para confidenciar suas experiências e tristezas, aumentando as chances de manifestar problemas emocionais, comportamentais e afetivos. Também, é importante salientar, que o suporte familiar seja eficaz de forma a garantir que o adolescente possa se desenvolver de forma mais equilibrada, sendo necessário que os relacionamentos dentro da família sejam plausíveis, uma vez que o desenvolvimento e a regulação emocional estão diretamente ligados aos vínculos familiares (Silva et al., 2011).

Outro resultado do estudo aponta que 47 dos 135 adolescentes, ou seja, 34,8\% da amostra, sente-se inútil e sem préstimo, dos quais 93,6\% possui percepção de suporte baixo e 6,4\% possui percepção de suporte alto.

Para Baptista, Alves e Santos (2008), crianças e adolescentes que viveram em famílias equilibradas, em que havia um clima de respeito mútuo, com poucos conflitos e relacionamentos familiares funcionais, tendem a desenvolver a crença de que possuem habilidades em lidar efetivamente com mudanças adversas na vida, quando comparadas com aquelas crianças e adolescentes que cresceram em um clima familiar com baixo suporte.

Sobre ideação suicida, 29 dos 135 adolescentes, ou seja, 21,5\% da amostra, apresentaram ideação, dos quais 89,7\% possuem percepção de suporte baixo e 10,3\% possui percepção de suporte alto. Sendo que recentes estudos trouxeram que o maior índice de suicídio de crianças e adolescentes está entre 15 e 19 anos. (Kõlves \& Leo, 2016b).

Couto (2017) observou em sua revisão que quando se trata de adolescentes e comportamento suicidas, a depressão foi o transtorno mais associado e, segundo os autores Kõlves e Leo (2016a), entre os anos de 1990 e 2010, no Brasil e em outros cinco países da América Latina, houve um aumento significativo do número de suicídios de adolescentes.

A prevalência da ideação suicida nos adolescentes está muito relacionada com a presença de depressão, consumo de álcool, tristeza, solidão, uso de drogas, falta de suporte e problemas de relacionamento com os pais. (Moreira $\&$ Bastos, 2015).

Os resultados encontrados apontam para uma associação positiva entre sintomas de sofrimento psíquico e percepção de suporte social. Na medida em que aumenta o escore do SRQ, maior o sofrimento psíquico, e quanto menor a pontuação no EPSUS-Ad, menor o suporte familiar percebido. Já se esperava correlações altas nos itens escolhidos, uma vez que os construtos envolvidos estão relacionados e, como trazido anteriormente, o suporte social é tido como aspecto protetivo para o desenvolvimento de transtornos mentais e em um menor sofrimento psíquico.
Os adolescentes com baixo nível de suporte familiar e social mostraram-se mais tristes, com sentimentos de inutilidade, ideação suicida e relataram chorar mais frequentemente. Esses dados enfatizam a conexão entre sistemas de suporte social e psicopatologia. Esses relacionamentos indicam que os jovens não sentem como se estivessem sendo cuidados e acolhidos por algum tipo de suporte e que isso favorece a presença de sofrimento psíquico e reforça a probabilidade de estes desenvolverem problemas psicopatológicos.

\section{Considerações finais}

Este estudo teve como objetivo determinar se há associação entre as variáveis estatisticamente significativas e determinar quais níveis de variáveis podem apresentar o maior impacto na associação. Com o presente resultado, confirmamos que há associações significativas e também uma associação indicando possibilidade de impacto da variável percepção de suporte sobre o surgimento de possíveis pródromos de transtornos mentais, principalmente de humor. Em uma pesquisa feita por Saffer, Glenn e Klonsky, (2015) com adolescentes, constatamos que os seus resultados corroboram com nossos achados, pois os mesmos verificaram que no grupo que relatou um menor índice de suporte parental havia histórico de tentativas de suicídio, quando comparado com o grupo de jovens com ideação suicida, mas sem tentativas.

Visto que os cuidados parentais e traumas precoces na infância devem ser considerados fatores de risco para o desenvolvimento de sintomas depressivos (Francke, Viola, \& Grassi-Oliveira, 2013; Ramos, 2018). Aliado a isso, entende-se que diferentes perspectivas do funcionamento familiar ou até mesmo a qualidade da relação entre os pais e os filhos interferem na qualidade da comunicação entre estes.

Importante ressaltar que este estudo está baseado em respostas subjetivas que passam pela percepção dos jovens respondentes, assim o que fica evidente é que estes jovens estão de alguma forma percebendo que os seus vínculos próximos não estão sendo suficientemente apoiadores para suas necessidades. Evans, Hawton e Rodham (2004) e Ramos (2018) já ressaltavam para a relação significativa da perspectiva de falta de apoio parental e conflitos familiares com desfechos de comportamentos de risco, como automutilação e tentativas de suicídio. E por último, sugere-se, através de políticas públicas de acesso universal e equânime, facilitar a interferência profissional, a fim de minimizar o sofrimento infantil/juvenil. Investindo na prevenção e promoção da saúde poderíamos contemplar uma perspectiva menos negativista destes jovens e ao mesmo tempo investir nos seus potenciais e atributos adaptativos, oportunizando o ajuste socioemocional das crianças e adolescentes em geral.

Entendemos que este estudo teve como limitação o fato de que a amostra foi restrita a uma única escola. Sugere-se que seja minimizada, ampliando a população pesquisada para amostras representativas de adolescentes das escolas municipais estaduais e particulares do município de Guaíba.

\section{Referências}

Alves, C. F., \& Dell'Aglio, D. D. (2015). Apoio Social e comportamentos de risco na adolescência. Psico, 46(2), 165-175. D0l: 10.15448/19808623.2015.2.18250.

Associação Americana de Psiquiatria - APA. (2013). DSM-5 - Manual Diagnóstico e Estatístico de Transtornos Mentais (5a ed.). Porto Alegre: Artmed. 
Associação Brasileira de Transtorno Bipolar - ABTB. (2016). Recuperado de http://www.abtb.org.br/transtorno.php.

Assumpção, A. A., Teodoro, M., Maltoni, J., \& Neufeld, C. B. (2017). A perspectiva adolescente na teoria cognitiva de Beck. In C. B. Neufeld (Org.), Terapia cognitivo-comportamental para adolescentes: uma perspectiva transdiagnóstica e desenvolvimental (Cap. 2, pp. 29-41). Porto Alegre: Artmed.

Baptista, M. N., \& Cardoso, H. F. (2011). Escala de Percepção do Suporte Social-Versão Infanto-Juvenil (EPSUS-Ad). Manual técnico não publicado.

Baptista, M. N., Alves, G. A. S., \& Santos, T. M. M. (2008). Suporte familiar, auto-eficácia e lócus de controle evidências de validade entre os construtos. Psicologia: Ciência e Profissão, 28(2), 260-271. Recuperado de http://pepsic.bvsalud.org/scielo.php?script=sci_arttext\&pi$d=\$ 1414-98932008000200004 \&$ lng =pt\&tlng=pt.

Baptista, M. N., Borges, L., \& Serpa, A. L. L. (2017). Gender and age-related differences in depressive symptoms among Brazilian children and adolescents. Paideia, 27(68), 46-53. D0l: 10.1590/1982-43272768201706.

Braga, L., \& Dell'Aglio, D. D. (2013). Suicídio na adolescência: fatores de risco, depressão e gênero. Contextos Clínicos, 6(1), 2-14. D0l: 10.4013/ ctc.2013.61.01.

Clemente, A. S. (2015). Concepções dos psiquiatras sobre o Transtorno Bipolar do Humor e sobre o estigma a ele associado. Tese de doutorado, Centro de Pesquisas René Rachou, Fundação Oswaldo Cruz, Belo Horizonte, MG, Brasil.

Couto, V. V. D. (2017). Comportamento suicida em adolescentes: prevalência e fatores associados. In Vínculo a pais e pares e comportamento suicida em adolescentes. Brasilia, DF: UnB. (No prelo).

Cruvinel, M.,\& Boruchovitch, E. (2014). Compreendendo a depressão infantil. Petrópolis, RJ: Vozes.

Evans, E., Hawton, K.,\& Rodham, K. (2004). Factors associated with suicidal phenomena in adolescents: a systematic review of population-based studies. Clinical Psychology Review, 24(8), 957-979. D0I: 10.1016/j. cpr.2004.04.005.

Ferrari, J., \& Barreto,\& J. B. M. (2018). Quando o viver não vale mais a pena: a percepção do paciente suicida. Pesquisa em Psicologia - Anais Eletrônicos, 171-198. Recuperado de https://portalperiodicos.unoesc.edu.br/pp_ae/ article/view/19204.

Francke, I. D., Viola, T. W., Tractembeg, S. G., \& Grassi-Oliveira, R. (2013). Childhood neglect and increased withdrawal and depressive severity in crack cocaine users during early abstinence. Child Abuse \& Neglect, 37(10), 883-889. D0l: 10.1016/j.chiabu.2013.04.008.

Gonçalves, M., Baptista, M. N.,\& Farcas, D. (2016). IPSF: análise da estrutura interna em uma amostra de jovens adultos portugueses. Avaliação Psicológica, 15(1), 115-123. D0l: 10.15689/ap.2016.1501.12.

Harding, T. W., Arango M. V., Baltazar, J., Climent, C. E., Ibrahim, H. H., Ladrido-Ignacio, L., \& Wig, N. N. (1980). Mental disorders in primary health care: A study of their frequency and diagnosis in four developing countries. Psychological Medicine, 10(2), 231-241. D0l: 10.1017/S0033291700043993.

Hay, W., Levin, M., Deterding, R., \& Abzug, M. (2016). Current pediatria: diagnóstico e tratamento. (22a ed.). Porto Alegre: AMGH.

James, S., Reddy, S. P., Ellahebokus, A., Sewpaul, R.,\& Naid00, P. (2017). The association between adolescent risk behaviors and feelings of sadness or hopelessness: a cross-sectional survey of South African secondary school learners. Psychology Health and Medicine, 22(7), 778-789. D0l: 10.1080/13548506.2017.1300669.

Kaplan, H. I., \& Sadock, B. J. (1993). Compêndio de Psiquiatria. Porto Alegre: Artes Médicas.

Kõlves, K., \& Leo, D. (2016a). Adolescent suicide rates between 1990 and 2009: analysis of age group 15-19 years worldwide. Journal of Adolescent Health, 58(1), 69-77. D0l: 10.1016/j.jadohealth.2015.09.014.

Kõlves, K., \& Leo, D. (2016b). Suicide methods in children and adolescentes. European Child \& Adolescent Psychiatry, 26(2), 155-164. D0l: 10.1007/ s00787-016-0865-y.
Lopes, C. S., Abreu, G. A., Santos, D. F., Menezes, P. R., Carvalho, K. M. B., Cunha, C. F., Vasconcellos, M. T. L., Bloch, K. V.,\& Szklo, M. (2016). ERICA: prevalência de transtornos mentais comuns em adolescentes brasileiros. Revista de SaúdePública, 50(1), 1s-9s. D0l: 10.1590/S01518-8787.2016050006690.

Moreira, L. C. 0., \& Bastos, P. R. H. 0. (2015). Prevalência e fatores associados à ideação suicida na adolescência: revisão de literatura. Psicologia Escolar eEducacional, 19(3), 445-453. D0l: 10.1590/2175-3539/2015/0193857.

Noto, M. N. V. N. (2014). Avaliação do período prodrômico do transtorno afetivo bipolar. Dissertação (Mestrado em Ciências) - Escola Paulista de Medicina, Universidade Federal de São Paulo, São Paulo, SP, Brasil. Recuperado de https://sucupira.capes.gov.br/sucupira/public/consultas/coleta/ trabalhoConclusao/viewTrabalhoConclusao.jsf?popup=true\&id_trabalho $=2134636$

Organização Mundial de Saúde - OMS. (2017). Depression and other Common Mental Disorders. Global Health Estimates. Genebra, Suíça: OMS. Recuperado de http://apps.who.int/iris/bitstream/handle/10665/254610/ WH0-MSD-MER-2017.2-eng.pdf;jsessionid=91AE8FEF5F91B201B0FD7562CE3964DD? sequence $=1$.

Phillips, M. L. \& Kupfer, D. J. (2013). Bipolar disorder diagnosis: challenges and future directions. The Lancet, 381(9878), 1663-1671. D0l: 10.1016/ s0140-6736(13)60989-7.

Pinheiro, M. N., Sousa, W. D. C., Feitosa, J. R. T., \& Batista, E. C. (2017). Identificação e compreensão de sintomas depressivos na infância em contexto escolar: desafios contemporâneos do educador. Revista Pedagógica, 19(40), 155-171. D0l: 10.22196/rp.v19i40.3748.

Ramos, V. A. B. (2018). Depressão na infância e adolescência. Psicologia.pt, 1-22. Recuperado de https://www.psicologia.pt/artigos/ver_artigo. php?depressao-na-infancia-e-adolescencia\&codigo $=\mathrm{A} 1224$.

Silva, I. R., Sousa, F. G. M., Santos, M. H., Cunha, C. L. F., Silva, T. P., \& Barbosa, D. C. (2011). Significados e valores de família para adolescentes escolares. Revista da Rede de Enfermagem do Nordeste, 12(4), 783-789. Recuperado de http://periodicos.ufc.br/rene/article/view/4340.

Saffer, B. Y., Glenn, C. R., \& Klonsky, D. (2015). Clarifying the relationship of parental bonding to suicide ideation and attempts. Suicide and Life-Threatening Behavior, 45(4), 518-528. D0l: 10.1111/sltb.12146.

Sampieri, R. H., \& Lucio, M.P. (2013). Metodologia de Pesquisa. Porto Alegre: Artmed.

Sette, C. P., Capitão, C. G., \& Carvalho, L. F. (2016). Depressive symptoms in patients with cancer. Open Journal of Medical Psychology, 5(1), 7-16. D01: 10.4236/ojmp.2016.51002.

Silva, L. L. T., Alvim, C. G., Costa, C. C., Ramos, T. M., \& Costa, E. E. (2015). 0 suicídio na adolescência nas publicações da enfermagem brasileira: revisão integrativa da literatura. Revista de Enfermagem do Centro Oeste Mineiro, 5(3), 1871-1884. D0l: 10.19175/recom.v5i3.767.

Souza, A. G., Barbosa, G. C., \& Moreno, V. (2015). Suicídio na adolescência: revisão de literatura. Revista UNINGÁ, 43, 95-98. Recuperado de https:// www.mastereditora.com.br/periodico/20150501_135302.pdf.

Souza, M. S., \& Baptista, M. N. (2017). Associações entre suporte familiar e saúde mental. Psicologia Argumento, 26(54), 207-215. Recuperado de https://periodicos.pucpr.br/index.php/psicologiaargumento/article/ view/19753

Vieira, G. P. M. (2015). Avaliação do suporte familiar em idosos: estudos de evidências de validade. Dissertação de mestrado, Programa de Pós-Graduação em Psicologia, Universidade São Francisco, Itatiba, SP, Brasil. 